\title{
Strengthening Health Surveillance through the Development of Interagency Relationships
}

\author{
Emily V. Glidden, Royal K. Law \\ HSB/ETT, CDC/ONDIEH/NCEH, Atlanta, Georgia, United States
}

Objective

- To discuss the development of a set of tools for interagency collaborations on health surveillance

- To determine the core contents of the tools based on known gaps in health surveillance

- To determine collaborators in development and timelines for completion

\section{Introduction}

In 2010, the Council of State and Territorial Epidemiologists (CSTE) identified data collected by poison centers (PCs) as an important tool for all- hazards exposure and illness surveillance. In response to this, the Centers for Disease Control and Prevention (CDC), CSTE, and the American Association of Poison Control Centers (AAPCC) members created the Poison Center Public Health Community of Practice (CoP). The CoP acts as a platform, to facilitate sharing experiences, identify best practices, and develop relationships among federal agencies, state and local health departments (HD), and PCs. Since its inception, the CoP garnered over 250 members, hosted more than 25 webinars regarding PC-HD collaborations, and produced five newsletters highlighting subjects pertinent to PC and HD personnel.

To date, the CoP's primary focus has been to strengthen PC-HD partnerships; however, recent events highlight opportunities to expand the public health impact of the CoP. In this roundtable, we will discuss how the CoP was leveraged by federal and state health agencies to build new multidisciplinary and inter-agency relationships and how these experiences have led to the proposed guidance. Some examples are summarized below.

In March 2018, the Illinois Department of Public Health (IDPH) responded to a cluster of synthetic cannabinoid exposures presenting to healthcare facilities with unexplained coagulopathy. The CDC's National Center for Environmental Health (NCEH) also identified the outbreak via routine surveillance activities using the National Poison Data System (NPDS). In response, NCEH enhanced their NPDS surveillance activities, to monitor for related clusters elsewhere, and facilitated information sharing between PCs, HDs and federal agency partners. NCEH requested that the CoP provide a national platform for NCEH and IDPH to disseminate information on the outbreak in a timely manner. Representatives of the Drug Enforcement Agency (DEA) and the Federal Bureau of Investigation (FBI) participated in the webinar and informed stakeholders about associated regulations and law enforcement efforts. NCEH was able to disseminate case definitions for public health surveillance as well as relevant points of contact for PC and HD staff to report suspected outbreak-related cases. Following the webinar, NCEH collected previously unreported, potentially outbreak-related cases. The webinar hosted 130 attendees (many reporting multiple participants per attendee record), largely due to the CoP's expansive network and helped enable information sharing, communication, and collaboration among national and state stakeholders.

Also in March 2018, the CoP hosted a webinar to inform members of the ongoing inter-agency public health efforts on HAB surveillance, toxicology, and regional protection efforts. Harmful algal blooms (HABs) are a growing public health problem and PC data collection is an invaluable health surveillance resource. In the webinar members from NCEH, the United States Environmental Protection Agency (EPA), National Center for Emerging Zoonotic Infectious Disease (NCEZID), and the Florida Department of Health all presented information about on their individual activities with HABs. The webinar discussions identified 1) shortfalls in current National Poison Data System (NPDS) exposure monitoring efforts for HABs and 2) a lack of familiarity with existing public health resources for state and local agencies to report exposures and access HAB subject matter experts (SMEs). As a result, NCEH HABs SMEs and AAPCC personnel have begun to help modify current NPDS HABs coding and develop new codes to bolster ongoing health surveillance efforts to improve HAB exposure monitoring.

ISDS Annual Conference Proceedings 2019. This is an Open Access article distributed under the terms of the Creative Commons AttributionNoncommercial 4.0 Unported License (http://creativecommons.org/licenses/by-nc/3.0/), permitting all non-commercial use, distribution, and reproduction in any medium, provided the original work is properly cited. 


\section{Description}

Findings and lessons learned from activities outlined in the introduction include the need for: 1) standardized inter-agency communication, 2) increased knowledge and utilization of state reporting and monitoring systems, and 3) inter-agency collaborations to prevent the duplication of efforts. In this roundtable, we will: 1) discuss how to develop information and tools for inter-agency public health communication and messaging, 2) identify key stakeholders including potential national, state, and local agencies who can help bolster communication messaging, and 3) develop appropriate points of contact within these agencies. Potential components of the guidance may include: 1) a comprehensive list of state resources available to PC and PH personnel, 2) recommended inter- agency points of contact, 3) lessons learned from collaborative projects, and 4) PC abilities to share and analyze data for public health practice and health surveillance. 